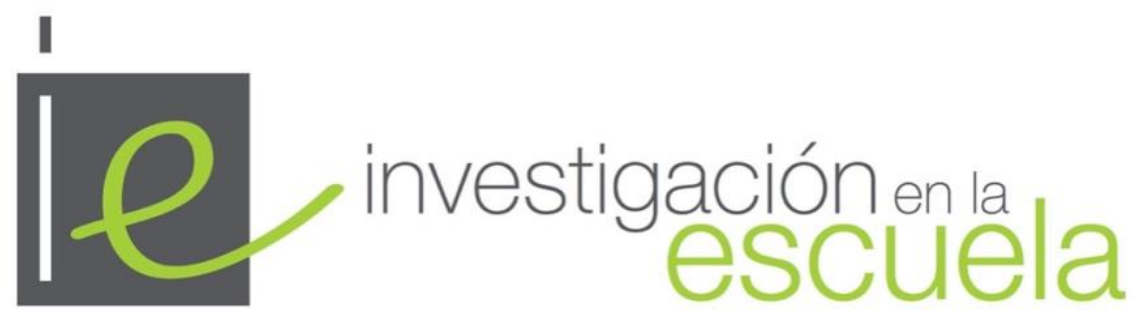

Revista de Investigación e Innovación Educativa nº 105, 2021 | e-ISSN 2443-9991

\title{
Estudio de casos e innovación educativa: un encuentro hacia la mejora educativa
}

\section{Case study and educational innovation: a necessary meeting for educational improvement}

Dra. Rosa Vázquez Recio, es Profesora Titular del Departamento de Didáctica en la Facultad de Ciencias de la Educación en la Universidad de Cádiz (España)·rmaria.vazquez@uca.es·Orcid https://orcid.org/0000-00016595-177X

Dra./Dr. Marina Picazo Gutiérrez, es Profesora Sustituta Interina del Departamento de Didáctica en la Facultad de Ciencias de la Educación en la Universidad de Cádiz (España) · marina.picazo@uca.es · Orcid https://orcid.org/0000-0002-0689-4573

Dra. Mónica López-Gil, es Profesora Ayudante Doctora del Departamento de Didáctica en la Facultad de Ciencias

iD de la Educación en la Universidad de Cádiz (España)·monica.maria@uca.es·Orcid https://orcid.org/0000-0002$5412-2244$

Cómo citar este artículo

Vázquez Recio, R., Picazo Gutiérrez, M. y López-Gil, M. (2021). Estudio de casos e innovación educativa: un encuentro hacia la mejora educativa. Investigación en la Escuela, 105, 1-10. doi: https://doi.org/10.12795/IE.2021.i105.01

Resumen. La innovación educativa, su significado y su necesidad es una tarea compleja en la medida en que su definición y su práctica están determinadas por el significado, enfoque adoptado y los propósitos que le damos a la educación. En un intento de facilitar esta tarea, se destacan dos posiciones de innovación. Por un lado, una perspectiva simplista, estrecha y mercantilista que responde a la ley de la oferta y la demanda. Por otro lado, una perspectiva crítica, ética y equitativa; en definitiva, una innovación con una visión humanista. Desde esta perspectiva, la innovación educativa se refiere a los cambios que experimentan las prácticas educativas para satisfacer las necesidades específicas y complejas del alumnado. Estas transformaciones deben ser entendidas desde los principios de justicia y equidad. No obstante, la innovación educativa forma parte también del discurso neoliberal y tecnocrático, al que define como productos y métodos replicables. Por lo tanto, entendemos la innovación educativa como una propuesta basada en la investigación y, concretamente, en los estudios de caso como una forma de hacer investigación situada. Estos ofrecen la posibilidad de alcanzar un conocimiento amplio y profundo de los problemas educativos particulares sin olvidar lo general, para llevar a cabo una innovación educativa: transformadora, emancipadora y profesionalizadora; contextual y original; comunitaria y colaborativa; procesual, impactante y relevante; de calidad y rigurosa.

Abstract. Educational innovation, its meaning and its need is a complex task where its definition and its practice are determined by the approach adopted, by the meaning and purposes that we give to education. In an attempt to facilitate this task, two positions of (educational) innovation stand out. On one hand, a simplistic, narrow and mercantilist perspective that responds to the law of supply and demand. On another hand, critical, ethical, and equitable perspective; briefly, an innovation with a humanist vision. From this perspective, educational innovation refers to changes that educational practices undergo to meet the special and complex student's needs. These socio-cultural transformations lived by the students must be understood from the principles of justice and equity. Nevertheless, educational innovation is part also of the neoliberal and technocratic discourse, which it defines as replicable products and methods.

Therefore, and after all that said, we understand educational innovation as a proposal based on research and, specifically, on case studies as a way of doing situated research. These offer the possibility to reach a comprehensive 
and deep knowledge of the particular educational problems. These offer the possibility to reach a comprehensive and deep knowledge of the particular educational problems, but without forgetting the common ones, to carry out an educational innovation: transforming, emancipating and professionalizing; contextual and original; communitarian and collaborative; processual, impacting and relevant; of quality and rigorous.

\section{Palabras clave $\cdot$ Keywords}

Innovación, estudio de caso, educación, mejora escolar, investigación educativa, mercado. Innovation, case study, education, school improvement, education research, market.

\section{Introducción}

Formamos parte de un tiempo que precisa de reflexión y de repensar lo que está ocurriendo a nuestro alrededor sin dejar fuera lo que se halla en los márgenes y más allá de estos. Son tiempos de cambios, pero también de constantes; cambios que, paradójicamente, están dejando intactos los elementos estructurales que generan desigualdad, inequidad e injusticia (constantes). Las políticas neoliberales saben manejarse bien en estas dos dimensiones, inyectando cambios que no dejan de ser un escaparate para preservar el mantenimiento de prácticas que no mejoran el bienestar ni miran al bien común. Estas políticas se han apropiado de un lenguaje (inclusión, participación, igualdad, autonomía, creatividad, innovación, etc.) del que solo han mantenido la "cáscara" para simular su compromiso con la ciudadanía en clave de justicia, democracia, libertad, y para disimular el contenido que remite a la ideología neoliberal, neoconservadora, capitalista, sostenedora de sistemas de dominación, marginación y desigualdad. Esta resignificación ha calado en todos los órdenes, incluyendo la educación, como no podía ser de otro modo, sobre todo cuando se reconoce que "la educación encierra un tesoro", como apuntara Delors en 1996.

Hemos adoptado toda una jerga de palabras que convergen en las claves del discurso hegemónico neoliberal: costes y beneficios, competitividad, rendición de cuentas, branding, excelencia, productividad, rentabilidad, etc., que se canalizan a través del currículum y los procedimientos de gestión y organización de los centros educativos. Hablamos de educación financiera, economía del conocimiento, economía de la felicidad, think tank... Formamos parte de un tiempo que precisa de reflexión.

En esta tarea de reflexionar y repensar, hemos puesto la mirada en la innovación educativa, no porque sea una acción novedosa, un "descubrimiento", sino, precisamente, porque también ha caído en la tela de araña del discurso neoliberal, instrumentalizándola y poniéndola al servicio de la economía del conocimiento - y de la llamada "robot economy" (Williamson, 2019). La innovación educativa necesita ser entendida desde una mirada no tecnificada sino dirigida, porque es posible, hacia la transformación y la emancipación. La existencia de prácticas educativas innovadoras debe ser visibilizada, pues desde su conocimiento se abre una vía que permite reconocer que, pese a los marcos impuestos por las políticas educativas neoliberales, es viable acciones alternativas y disidentes. Llegar a esas prácticas es posible a través de la investigación, y entre las diversas formas de investigar, entendemos que los estudios de caso es una opción coherente con las premisas en las que se sustenta una innovación educativa comprometida con la inclusión, la justicia y la equidad.

En lo que sigue se desarrolla este planteamiento, comenzando por el estudio de caso y sus particularidades, para pasar a concretar el sentido de la innovación educativa, dejando marcado cómo debe ser esta para no ser convertida en una mercancía más del sistema educativo. Desde estos dos territorios, se teje el encuentro necesario entre el estudio de caso y la innovación para la mejora educativa.

\section{El estudio de caso y la revelación de mundos}

Estar en el mundo es una acción necesaria pero no suficiente si queremos ser algo más que sujetos que suman para ese todo que lo configura. Estar precisa de acciones como ser, pensar, sentir, dialogar, etc., que convergen en el camino hacia la comprensión de ese mundo con el que mantenemos una ligadura que nos atraviesa. Dicha ligadura genera la pertenencia a ese mundo, y, al mismo tiempo, a sus territorios y también a sus márgenes. El mundo al que pertenecemos no es un mundo que se agote en sí mismo, sino que acoge en su seno la riqueza de mundos posibles y realidades disponibles, esto es, diferentes formas de estar, de conocer, de experiencias, relaciones, saberes, sentires, miradas, etc. La comprensión pretendida de ese mundo, de sus mundos posibles y realidades disponibles encuentra en los estudios de caso su aliado idóneo, porque en este se conjugan dos componentes esenciales: la singularidad y la complejidad.

Los estudios de casos han pasado la frontera, abandonando el territorio de las consideradas investigaciones menores, una forma de investigar de menor alcance, de inferior rango científico, con dificultades para garantizar fiabilidad y validez - que no son, precisamente, los criterios propios para 
determinar su calidad (Flick, 2014), junto con otras atribuciones que simplifican y/o distorsionan el sentido de lo que es investigar con estudio de caso, como es solo realizar observaciones, estar mucho tiempo en el campo, recopilar datos, etc. Esta desvalorización o merma del potencial de los estudios de caso puede derivarse del hecho de entender que su propósito se centra en el estudio de un solo caso (Verschuren, 2003). La literatura disponible revela la consolidación de los estudios de caso como una forma de hacer investigación legítima (Hammersley y Atkinson, 1994; Mills et al., 2009; Ragin \& Becker, 1992; Simons, 2011; Stake, 1998, 2006; Stoecker, 1991; Travers, 2006; Walker, 1983, 1989; Yin, 2004, 2011, 2018) con principios y criterios diferentes de los que rigen las investigaciones en ciencias experimentales (Sthenhouse, 1987, p. 21). No obstante, ese abandono no ha conllevado un reconocimiento efectivo. Más allá de lo que puede significar esa explicación del interés en solo un caso - por otra parte, reduccionista y cercano a lo que ya apuntasen Campbell y Stanley (1995) al considerar que los estudios de un solo caso son poco rigurosos y científicos-, los motivos que llevan a deslegitimar a los estudios de caso como una forma de hacer investigación, con entidad en sí misma, son más profundos, y se sitúan en el debate de la supremacía de la investigación cuantitativa, a la que se la reconoce como la auténtica, la legítima y la autorizada para explicar los fenómenos sociales con rigor científico. Este reconocimiento se sostiene en el que viene siendo el pensamiento hegemónico en investigación educativa sustentado en el positivismo. "Malos tiempos" son para la investigación cualitativa, y en concreto, para los estudios de caso, porque nos encontramos en un momento en el que interesa la investigación basada en la evidencia (Biesta, 2007; Elliot, 2001; Hammersley, 2001): "evidencias científicas" que solo pueden derivarse de "investigaciones científicas" en las que el "método científico" es el que marca la pauta. En el fondo se apuesta por la tecnificación y cuantificación como garante de la eficacia y eficiencia, la rendición de cuentas, la validez en la educación (Oliver \& Conole, 2003), la imposición de criterios estandarizados para definir la calidad de las investigaciones y la regulación del mercado cognitivo que impide el logro de la justicia cognitiva, y, consecuentemente, de la justicia social (Santos, 2009, 2010, 2019). Ante tales circunstancias, los estudios de caso, y formas de investigación afines, quedan invisibilizados, ensombrecidos o relegados a la consideración de estrategias de investigación (Verschuren, 2003) (que no forma de hacer investigación en sí misma) "auxiliares", es decir, con función complementaria a estrategias cuantitativas. De esta forma, lejos queda hacer investigaciones que tomen a los estudios de caso como forma única de hacer investigación, con toda la complejidad que encierra como proceder indagatorio.

Asumiendo las fisuras y las dificultades de contar con una única definición (que no tiene que ser per se una limitación), el estudio de caso es una forma de investigar la realidad ("caso") de un modo que nos permite alcanzar un conocimiento profundo, que llega a ser tal porque se adopta un análisis exhaustivo en el que se combina lo singular, lo idiosincrásico, lo único - aquello que lo hace intransferible, legítimo en sí mismo y clave para su propia concreción- con la complejidad que caracteriza a toda realidad ("caso") encarnada en el contexto que le es propio, y cuyos límites que lo separan de este no siempre están claramente definidos (Simons, 2011, p.41). Podemos decir que "cada caso es un mundo" singular y complejo.

Stenhouse (1978, p. 31) se refería al estudio de un caso en progreso, en curso, en acción: una "vida" para ser contada, narrada. En palabras de Walker (1983, p. 45), "el estudio de casos es el examen de un ejemplo en acción. El estudio de unos incidentes y hechos específicos y la recogida selectiva de información de carácter biográfico, de personalidad, intenciones y valores, que permite al que lo realiza, captar y reflejar los elementos de una situación que le dan significado", siendo mensurable lo idiosincrásico y lo singular como legítimo en sí mismo. Paralelamente, el conocimiento comprensivo y profundo de la realidad puede constituirse en fuente de ideas para pensar e interrogarnos por problemas más amplios que transcienden el propio caso, pero lo hacemos desde el conocimiento que nos ofrece el mismo (Walker, 1983; Stake, 1998; Yin, 2011, 2018). En este conjunto de consideraciones, asumir esta forma de investigar es adquirir un compromiso ético-político que ayude a revelar las desigualdades y las injusticias para la consecución de una educación democrática e inclusiva.

Los estudios de caso los asumimos como esa forma de hacer investigación en educación que nos ofrece la posibilidad de llegar a un conocimiento comprensivo y profundo de las escuelas, las prácticas docentes, la micropolítica escolar, el currículum, las prácticas inclusivas e innovadoras, etc., aspectos, todos y otros por añadir, que no dejan de ser mundos ("casos") revelados gracias a los estudios de caso como forma de hacer investigación situada.

\section{La innovación educativa, la alteración como estilo educativo}

Una simple mirada a nuestro alrededor nos hace percatarnos de las constantes y profundas transformaciones sociales, culturales, económicas y tecnológicas que venimos viviendo. La "nueva normalidad" es ya un hecho, pero lo cierto es que vivimos en constante renovación (o, al menos, 
adaptación). Los cambios, de hecho, forman parte de nuestra cotidianeidad y hoy más que nunca todo resulta inestable, beta, caduco, finito, inmediato y líquido (Bauman, 1999). La necesidad de mejora continua de la escuela incita a pensar que los cambios forman parte de su esencia. Si bien para mejorar hay que generar cambios, no todos los cambios implican mejoras, lo que nos insta a pensar que no todo cambio es válido ni adecuado.

La innovación es un término presente en los discursos de ámbito económico, cultural, político y, cómo no, educativo, y es presentada como la estrategia de mejora y la más adecuada para enfrentarse con éxito a los cambios y a las transformaciones para cubrir las nuevas y complejas necesidades que se originan. Nos estimula, precisamente, a cambiar nuestras prácticas docentes, pues el cambio provoca otro/s cambio/s. De hecho, la innovación se presenta como uno de los ejes prioritarios en las políticas educativas nacionales e internacionales con el fin de satisfacer las nuevas necesidades socioculturales y profesionales. No obstante, debemos advertir su carácter polisémico.

Abordar reflexiones sobre qué es innovar (en educación) y qué sentido tiene hacerlo resulta complejo, y su definición y su práctica devienen por el enfoque adoptado, por el sentido y los fines que le otorgamos a la educación y por los valores sociales imperantes, más vinculados a una perspectiva mercantilista y afín a cómo se afronta desde sectores empresariales, de respuesta a la ley de la oferta y la demanda o más ligado a una perspectiva crítica, ética y equitativa; en definitiva, una innovación con visión humanista (no reducida a las tecnologías).

Si bien el concepto de innovación que tuvo su origen en el ámbito de la industria ha sido adoptado en el contexto educativo desde la década de los 80 debido principalmente a los trabajos de Huberman, Fullan o Havelock, hace unos años que se viene relacionando íntimamente con la sociedad de consumo en la que estamos inmersos (y la refleja). Así, son numerosos los productos y los programas que se presentan con carácter educativo y que se ofertan (y se venden) como innovadores, y se procede de tal manera para responder a las necesidades educativas del alumnado: metodologías encapsuladas y transferibles, instrumentos, recursos, aplicaciones, etc.; programa y productos con los cuales pretenden, precisamente, mantener el orden tecnocrático a modo de métodos replicables, entre otros.

Tal es así que son muchas las entidades no educativas (empresas, fundaciones u organizaciones) que vienen ofreciendo propuestas de lo que asumen como innovaciones educativas -sin serlo en el fondo-, tales como Microsoft, Fundación la Caixa, Fundación Telefónica, Aulaplaneta, Atresmedia, Fundación Amancio Ortega, etc. Todos ellos ofrecen soluciones mágicas (y privadas) a las complejas problemáticas del campo educativo y prometen acabar con el fracaso educativo o las deficiencias formativas del profesorado. Unas innovaciones que deben ser financiadas por las propias escuelas (o por estas grandes empresas sin tener muy claro el coste que supone) y que hacen dudar al profesorado en activo de su profesionalidad y de su formación desde la lógica de la simplicidad, la técnica y la irreflexión. Y tal es el boom de las ofertas innovadoras para la educación desde ámbitos no educativos que, dentro de la cultura del ansia, de lo caduco, de lo medible o de la acumulación, la innovación resulta imperativa, que no nacida.

El problema es que estos productos y propuestas supuestamente innovadoras (y educativas) parchean las problemáticas que pretenden solventar, suponiendo meras apariencias o, en el mejor de los casos, un cambio de prácticas ofertadas desde la privatización educativa con un enfoque más empresarial que de mejora continua o de a largo plazo y que se justifican desde la necesidad de la digitalización (que no digitalidad) ${ }^{1}$ de la escuela o del desarrollo de la cultura del emprendimiento y el talento con las críticas que podríamos hacer a ello. Esencialmente no suponen cambios relevantes en pro de una educación más humana, más justa y más equitativa que cumpla con la función educativa de la escuela. No podemos negar que generan beneficios, pero estos son más de índole económico (a quienes las han creado) que educativo y, por tanto, en muchas ocasiones acaban originando desigualdad educativa y social.

Y es que la innovación educativa debe ser, cuanto menos ética (Harris, 2007). Desde este enfoque entendemos que la innovación educativa como estrategia de mejora educativa debe generar bienestar y equidad, constituir oportunidades de participación activa y distribuida de la comunidad educativa y posibilitar el desarrollo profesional del profesorado.

Puesto que la innovación está presente en los discursos y en las políticas educativas actuales, como ya se mencionó, incluir el análisis del contexto político como elemento que apoya o limita la innovación educativa y el sentido en el que lo hace nos resulta interesante.

Promover la innovación educativa es una de las tareas que se atribuían a la dirección de los centros educativos en la LOE, la LOMCE y se mantiene vigente en la LOMLOE, otorgándole un claro carácter jerárquico y tecnoburocrático como instrumento (que no estrategia) para el incremento del rendimiento académico (que no necesariamente de la calidad educativa). No obstante, la innovación durante la LOMCE

${ }^{1}$ Para mayor información sobre digitalidad versus digitalización véase López-Gil y Bernal Bravo (2016). 
se ha presentado como algo anecdótico, extraordinario y desde una perspectiva estrecha de su sentido. Solo se mencionaba en cuatro ocasiones ( $y$ una de ellas es para hacer alusión a un objetivo que no es propio de esta ley, frente a otros como evaluación (164), rendición de cuentas (10) o esfuerzo (9). La escasa presencia del concepto y de la estrategia de la innovación educativa en esta ley fue reflejo de la poca relevancia que se le otorgó en un sentido crítico. No obstante, se mantuvieron algunas propuestas de innovación que ya se promulgaba en la LOE, en la que, precisamente, la innovación educativa suponía uno de sus principios educativos: "el fomento de la promoción de la investigación, la experimentación y la innovación educativa", y promovía el fomento de los programas de investigación e innovación (art. 102) considerándose mérito a tener en cuenta a efectos de concursos de traslado y promoción.

No en vano se abrieron y actualizaron convocatorias como la reciente Resolución de 20 de diciembre de 2019, de la Dirección General de Formación del Profesorado e Innovación Educativa, por la que se efectúa la convocatoria de medidas de apoyo, aprobación y reconocimiento al profesorado para la realización de proyectos de investigación, e innovación educativa y de elaboración de materiales para el curso académico 2020/2021 y que viene a mantener la convocatoria de la Orden de 14 de enero de 2009 por la que se regula las medidas de apoyo, aprobación y reconocimiento al profesorado para la realización de proyectos de investigación e innovación educativa y de elaboración de materiales curriculares, en las condiciones que figuran en dicha orden.

Estas convocatorias vienen a presentar una visión estrecha y tradicional de la innovación, en tanto que no suponen un cambio auténtico (entendido como cambio trascendente y duradero) de la práctica educativa para la mejora y el aprendizaje, ni como parte consustancial de la profesionalidad docente. Por un lado, se presenta como incentivo, externo al profesorado, y, por otro, limitante en cuanto al ámbito de aplicación (suele coincidir con las materias STEM y la cultura emprendedora) que no necesariamente responde a las necesidades reales de los centros.

La LOMLOE vuelve a tomar con fuerza el concepto de innovación estando presente en más de trece ocasiones, vinculándolo de forma constante con la formación permanente y la posibilidad de aprendizaje. De hecho, se retoma la tarea del fomento de la creación de grupos de investigación e innovación educativa con el fin de mejorar las prácticas docentes y los procesos educativos, elevar los resultados y asegurar la calidad de la educación con mayor equidad e inclusión desde el intercambio de los resultados relevantes de la investigación e innovación educativas entre redes de centros educativos y las universidades (Disposición adicional trigésima quinta).

Asimismo, se recoge como base fundamental de la formación permanente del profesorado la necesidad de impulsar el trabajo colaborativo y las redes profesionales y de centros para el fomento de la formación, la autoevaluación y la mejora de la actividad docente a través de la formación en investigación e innovación educativa (art. 102, LOMLOE).

Esto supone que la innovación educativa es mucho más que la creación (o aplicación) de materiales y recursos de forma irreflexiva y descontextualizada de productos acabados. Es una cualidad y una aptitud como rasgo definitorio del profesorado y de la cultura escolar determinadas por la búsqueda constante de estrategias que respondan a un contexto cambiante con necesidad de conocimiento y que altera, de alguna forma, el orden anteriormente establecido. Pero además de conllevar en cierta forma una metamorfosis e implicar modificaciones en los recursos, en los principios pedagógicos o en las estrategias didácticas, transforma a las personas, a saber, a las que realizan los cambios y a aquellas a las que repercuten estos (aunque podríamos cuestionar esta distinción, ya que quienes idean y ejecutan la innovación se ven siempre afectados por ella).

Abogamos por una innovación como estrategia de mejora de la calidad educativa originada desde dentro de las escuelas, que no es impuesta sino una actitud constante (García Gómez, 2006) fruto del consenso y de la colegialidad, pensada para el presente y, por tanto, contextualizada, que viene a alterar las dinámicas tradicionales en pro de priorizar las situaciones susceptibles de mejora o resolución.

La innovación educativa desde estos parámetros será definida por ser:

- Transformadora: Implica la transformación (que no la mera incorporación) de recursos, metodologías, aspectos organizativos u otro elemento de los procesos de enseñanza y aprendizaje que huya de concepciones tradicionales de la educación de carácter tecnocrático hacia espacios de aprendizaje cooperativos, activos, dialógicos y equitativos.

- Emancipadora y profesionalizante: la escuela sin prescripciones externas toma decisiones sobre sus innovaciones desarrollando su ejercicio de autonomía, lo que debe conllevar al desarrollo profesional del docente, de la comunidad educativa y, por ende, de la educación. Supone un reconocimiento de la profesionalidad docente y de una comunidad educativa autónoma, flexible y democrática. Una innovación de abajo a arriba, ideada, liderada y desarrollada por el profesorado (pero que puede contar con agentes externos) basada en la profesionalidad reflexiva y crítica. 
- Contextual, original e inédita: estas transformaciones serán válidas solo para un contexto y momento específico.

- Comunitaria, colectiva y colaborativa: unos cambios que derivarán y se proyectarán desde el sentido de lo común y para el bien común.

- Procesual, perenne, adaptativa: alejada de modas y ensoñaciones y cercana a ser una actitud analítica para la comprensión de la casuística de los procesos de enseñanza y aprendizaje, y de alerta de lo que debe preservarse, modificar, suprimir, mejorar... en constante proceso de aprendizaje, centrándonos más en los procesos del cambio que en los contenidos del cambio (Milles, 1964).

- Impactante: genera efectos que se esperan que sean positivos.

- Relevante: en cuando que los cambios deben responder a las necesidades reales de ese contexto.

- De calidad: entendiendo calidad como transformaciones que respondan a la humanización de la educación, sostenibilidad y desarrollo profesional más allá del incremento de resultados académicos.

- Rigurosa: fruto de la investigación como proceso de profundización del conocimiento de la situación educativa susceptible de mejora y así otorgarle rigurosidad y coherencia. Del mismo modo, la investigación ayudará a la validación de la innovación creada a través del análisis del impacto de los cambios realizados y sobre si efectivamente se ha logrado la mejora colectiva y el camino hacia la justicia social y curricular.

La innovación como proceso que requiere el análisis de en qué punto está la escuela y hacia dónde quiere dirigirse, exige recurrir a la reflexión y a la autoevaluación de la propia práctica individual (cada docente hacia sus grupos-clase) y conjunta (el profesorado responsable de un grupo clase/claustro de un centro), así como a un pensar de manera crítica y dialógica (Canabal et al., 2018; Giroux, 2013; Zeichner y Flessner, 2009). Se asume que "el proceso de comprender y perfeccionar el propio ejercicio docente ha de arrancar la reflexión sobre la propia experiencia" (Zeichner, 1993, p. 45) y, por ende, la investigación resulta imprescindible, pero eso sí, no cualquier forma de hacer investigación. Será aquella cuyos resultados no propondrán innovaciones ideales, extrapolables y generalizadas sino resultados idiosincráticos que requerirán innovaciones particulares no empaquetadas en manuales de instrucciones para su aplicación. Así la calidad de los cambios innovadores aplicados no derivará de estándares que cuantifican la realidad, sino de la idoneidad de los mismos propuestos por la comunidad donde se genera y en pro de la equidad de sus miembros.

Una investigación que a tenor de que lo que se busca es la mejora de un colectivo concreto bajo el marco de la equidad y la inclusión, con rasgos identitarios muy específicos, requerirá de metodologías de investigación sensibles al contexto único con fines relacionados con el conocimiento profundo de los procesos formativos, el análisis y la reflexión como son el método etnográfico, los estudios de caso o la investigación-acción.

En síntesis, optar por una visión de la innovación educativa alejada de modas y posturas tecnocráticas requiere de un compromiso para con la educación, adoptar una postura consciente y de conciencia colectiva sobre las necesidades de las personas que conforman la escuela, aportando cambios profundos y fundamentados más allá de lo normativo hacia una educación más justa, más emancipadora y más humanizada.

\section{Los estudios de caso como aliado para la innovación educativa}

Desde el recorrido trazado, cabe preguntarse por las posibilidades de vincular los estudios de caso y la innovación educativa. ¿En qué medida esta forma de hacer investigación se hermana con solidez con la innovación educativa entendida en los términos expuestos?

Partiendo de una reflexión pedagógica y en un intento de discernir las características propias de lo que entendemos como innovación educativa en contextos particulares, como hemos mostrado anteriormente, y más allá de los parámetros establecidos que nos ofrecen la posibilidad de separar, en una primera criba, lo que es paja de aquello que es trigo, no podemos negar que nos encontramos ante un escenario que nos hace bucear en un mar de dificultades o, cuanto menos, de aprietos, emanados de la propia idiosincrasia del término, de los objetivos que se plantea y, por supuesto, de su puesta en práctica. Pero a su vez, y sin ánimo de caer en la incongruencia, dentro de este océano de particulares emergen determinadas certezas que se articulan en torno a un modo de pensar, hacer y entender la educación, en donde la "innovación" no queda establecida por una palabra que se agrega al sujeto, ni como una mera acepción declarativa, es decir, no podemos describirla por una serie de ítems que se generalizan para todas las acciones llevadas a cabo bajo este rótulo.

Desde esta mirada, buscamos profundizar no solo en las razones de la innovación educativa en la práctica a partir de los contextos particulares que nos proporcionan el aula y la escuela, sino también en el 
sentido propio de la innovación como un proceso social y colaborativo llevado a cabo desde el compromiso docente que nace de la investigación sobre la práctica (Cañal, 1987; Stenhouse, 1984). Partiendo de los referentes que delimitan la innovación educativa, apartándose de ser un mecanismo más puesto al servicio del mercado educativo, cabe insistir que la innovación educativa debe ser definida por el propio contexto en donde se desarrolla, así como su temporalidad o la consecución de sus objetivos de novedad o reforma, pudiendo ser y dejar de ser. Por tanto, el pretendido cambio educativo requiere de la búsqueda de un proceso de mejora, pero no como causa lógica de un devenir de acciones originales y creativas y hasta novedosas y diferentes (que también), sino como resultado de un proceso intencionado caracterizado por dar nuevas respuestas a los problemas y necesidades reales existentes en la práctica y en donde no todo está trazado y preestablecido.

Lo dicho hasta el momento nos lleva a la necesidad de subrayar el proceso y la experiencia vivida sin menos cabo de la planificación, pero alejados de modelos generalizables y homogéneos, como elementos que coadyuva en esta transformación inherente al concepto de innovación educativa desde sus inicios. Y es en este proceso realizado dentro de la innovación educativa en donde se hace necesario recurrir al estudio y a la investigación de experiencias que nos hagan pensar y analizar no tanto los resultados, sino cuestiones que nos inviten a pensar sobre el cuándo, cómo, dónde o por qué de la singularidad, dejando a un lado la generalidad de un modelo obsesionado como el producto final del mismo. Es decir, no vale con reducir el análisis a ejemplos homologables a cualquier generalidad, pues en educación queda ampliamente demostrado que ni los primeros aportan herramienta de valor, ni la generalidad permite comprender en profundidad los procesos de cambio reales que se dan en la práctica de las escuelas. Es por ello por lo que el valor de la investigación como proceso de reflexión sobre la práctica individual y singular se vislumbra imprescindible y necesario para poder llevar a cabo un análisis concienzudo, exhaustivo y comprensivo de la realidad y de su complejidad. Pero, además, esta investigación debe de permitir un conocimiento profundo de lo idiosincrático y particular, al mismo tiempo que discernir sobre el propio procedimiento, los cambios llevados a cabo, las transformaciones y mejoras producidas en la calidad educativa, así como en su rigurosidad y coherencia.

Por las propias características citadas, no cualquier forma de hacer investigación resulta ser un enfoque apropiado para llevar a cabo este tipo de análisis. No podemos olvidar que nos encontramos ante un estudio que requiere de una metodología que busca centrarse en lo característico y específico de ella, pero sin olvidar la importancia de lo común, de lo complejo del mismo. Todo ello con la intención de ir más allá de la experiencia descrita, para así dar respuestas a problemas que favorezcan la creación de una sociedad más justa a través de nuevas formas de sentir, pensar y hacer en educación. De entre las posibles formas de investigar que pueden satisfacer estos propósitos, entendemos que los estudios de caso pasan a ocupar un lugar destacado.

Llegados a este punto, cabe preguntarnos cómo el estudio de caso nos puede ayudar a abordar la innovación educativa desde una perspectiva más amplia que nos permita analizar experiencias innovadoras de éxito orientadas a la transformación educativa con equidad. Todo ello bajo el propósito de mejorar de forma efectiva la enseñanza, alejándonos de aquellas acciones que bajo el eslogan de "innovación" no dejan de ser ejercicios que entrañan cierto interés por introducir cambios curriculares o herramientas novedosas en cuanto a estrategias, organización o recursos, entre otros, pero que se quedan en un cambio superfluo para el que no se plantea el porqué del mismo o su propia finalidad.

Vázquez Recio y Angulo Rasco (2003) ofrecen una serie de rasgos y características en las investigaciones con estudios de casos que nos desvelan el gran valor de esta forma de investigar en la innovación educativa. Así pues, y siguiendo la línea que ya esbozó Simons (1996), más allá del propio concepto, el estudio de caso ofrece una serie de herramientas que lo hacen especialmente apto sobre otras metodologías y que procura un análisis profundo y concienzudo de la innovación educativa y sus consecuencias en la práctica, su vinculación con el contexto y sus efectos en el tiempo.

El estudio de caso nos conduce a la particularidad de los escenarios concretos, de manera que permite acercarnos al cambio y a la mejora educativa desde el propio caso (realidad). Ofrece la oportunidad de investigar y aprender de aquellas experiencias innovadoras que emergen como espacios significativos cargados de conocimiento práctico basado en la realidad existente de un determinado contexto. A partir de lo individual, de lo único y específico, permite dirigir nuestra mirada hacia aquellas experiencias que presentan interés por su efecto en la mejora de la enseñanza desde el principio de justicia social, equidad e inclusión existente dentro de la innovación educativa como práctica social, crítica y política, y no como tendencia hegemónica centrada en la estandarización. Encontramos trabajos que ilustran lo expuesto, como el elaborado por Rodríguez Martínez y Vázquez Recio (2002) en su aproximación a la escuela rural de Bolonia. 
Esta investigación nos proporciona la posibilidad de acercarnos a la realidad dentro de un contexto determinado desde un estudio de caso. Es a partir de las entrevistas elaboradas en el propio centro, el análisis documental y la observación, desde donde se nos ofrece la posibilidad de aprender de este caso. Lo particular del mismo nos lleva a entender los cauces seguidos dentro de la búsqueda de la mejora de la enseñanza y, por supuesto, nos brinda la posibilidad de acercarnos a lo singular, y es a partir del mismo desde donde se nos proporcionan ejemplos sobre los que construir conocimiento.

Si revisamos el trabajo realizado, además, podemos observar cómo la capacidad que confiere a la investigación el poder formar parte de las vivencias de las personas implicadas, mediante la escucha de sus historias, de sus interrogantes, aspiraciones e incertidumbres, permite a las investigadoras determinar otros muchos aspectos relacionados, por ejemplo, con el contenido escolar y su función social o con el trabajo autónomo y responsabilidad del alumnado, así como la formación docente que se sigue para llevar a cabo las experiencias descrita. Así pues, a través de la investigación podemos llegar a conocer las satisfacciones y controversias que la descrita experiencia genera dentro de las personas implicadas, o el grado de sostenibilidad que se observa para construir una nueva cultura docente, entre otras muchas.

En un segundo lugar, y no enumerado de forma jerárquica, el estudio de caso obliga, para tener en cuenta lo común, que, según Stake (1998), no solo hace referencia a otros contextos sino también a otros casos con los que surgen puntos de encuentro. Los estudios de caso de innovación educativa proporcionan la posibilidad acercarnos a la estructura organizativa del propio caso, el diseño curricular, la importancia de los recursos digitales utilizados, la implicación de las familias, la propia intención de llevar a cabo una propuesta de mejora, y un largo etc., Todas estas cuestiones nos describen rasgos y características que pueden ser propias o compartidas por diferentes proyectos innovadores, y que nos permiten observar lo convencional y lo inusual de cada caso investigado. Así pues, siguiendo con los estudios de caso realizados sobre la escuela rural de Bolonia, estos dieron lugar a nuevas investigaciones que focalizaron sus objetivos en la evaluación y análisis del uso de las tecnologías móviles en los procesos de enseñanza y aprendizaje de escuelas de diferentes países (España y Grecia). Angulo et al. (2005) se interesaron por el impacto, dentro de la escuela rural de Bolonia, que las tecnologías móviles pueden llegar a tener en el proceso de enseñanza y aprendizaje del alumnado que las usa. Desde esta perspectiva y utilizando el estudio de casos como instrumento de análisis para llegar a una mejor comprensión de la realidad, se nos muestra qué rasgos son propios de un ambiente determinado y cuáles son susceptibles de ser propios de varias culturas o contextos. Así pues, observamos cómo el estudio de caso también define una relación entre partes de una totalidad. Es decir, nos permite observar analogías que nos pueden ayudar a identificar determinados factores que pueden estimular la innovación en los centros educativos.

Pero, además, la ventaja que proporciona los estudios de caso para profundizar y conocer las razones de la innovación educativa, su sentido y su finalidad, reside en la flexibilidad que posee para su adaptación a las circunstancias y al medio. De manera que este ofrece la posibilidad de reajuste a las desviaciones o los cambios dentro de la propia investigación. Esta cuestión emerge como fundamental, pues ofrece la oportunidad de estudiar la innovación educativa fuera de los límites espacio temporales del momento concreto (Simons, 1996). Cuestión esta fundamental si entendemos la innovación educativa como un proceso de cambio y mejora procesual, perenne y adaptativo llevado a cabo dentro de un contexto educativo cambiante.

Desde esta perspectiva, Vázquez Recio (2011), años después y partiendo de los estudios anteriores realizados, ofrece una nueva visión de la escuela rural de Bolonia desde la enseñanza para la comprensión y de la experiencia educativa de la misma. En este trabajo refleja las inmensas posibilidades de flexibilidad y adaptación que proporciona el estudio de casos para analizar y dar voz al proceso de innovación y los cambios producidos en un largo periodo de tiempo dentro de un mismo caso.

Pero este acercamiento a la particularidad del propio caso, también nos acerca a las personas protagonistas de ellos. En este sentido, es indudable que en cualquier proceso de cambio educativo se hace necesario dar la palabra al profesorado, que es el grupo central de un proyecto de cambio y es este el que debe de explicar y, por tanto, debemos conocer sus patrones interpretativos que, como bien sabemos, no siguen obligatoriamente unas pautas lineales y que nos hablan de cómo mejorar lo que ocurre en la práctica (Martínez Bonafé, 2021). Así pues, el estudio de casos responde a esta necesidad descrita de la comprensión de la realidad social, entendida como una realidad cambiante y dinámica, no solo desde el contexto sino también desde las personas protagonistas del mismo, los trabajos realizados por Sego et al. (2017), Losada Iglesias et al. (2017), Iglesias Martínez et al. (2018), cuyos objetivos se focalizan en conocer la opinión del profesorado en proyectos de innovación educativa llevados a cabo desde la práctica docente.

Cuestiones todas estas que no hacen otra cosa más que poner de relieve la importancia de la innovación educativa apoyada en la investigación con estudio de caso. Una investigación que permita reflexionar y adecuar la enseñanza a los desafíos que la nueva realidad social y cultural plantea con objeto de asegurar que 
la calidad educativa alcance a todos los escolares para contribuir al desarrollo de una sociedad más justa, democrática y equitativa

\section{A modo de cierre y nuevas aperturas}

El recorrido realizado ha permitido poner de manifiesto no solo la posibilidad de vincular los estudios de caso con la innovación educativa, sino también, y, sobre todo, la pertinencia de hacerlo para poder promover acciones para la mejora educativa, dirigidas a la inclusión, la equidad y la justicia. El conocimiento de prácticas educativas innovadoras, en su sentido no tecnificado y mercantilizado, se convierten en una oportunidad para hacer posible una educación que garantice una respuesta coherente, ajustada y precisa a las necesidades que presenta el alumnado al que acoge. Esta propuesta desarrollada representa la apertura a nuevas vías de acción en las que investigación e innovación educativa comparten el propósito de mejorar la educación bajo el principio del bien común.

\section{Referencias}

Angulo, J. F., Betanzo, M. J., y López, M. (2005). Viviendo los contenidos: una experiencia en el uso de tecnologías móviles en los procesos de Enseñanza y Aprendizaje. Quaderns Digitals, 37. https://bit.ly/3sNh4OY

Bauman, Z. (1999). Modernidad Líquida. Fondo de Cultura Económica.

Biesta, G. (2007). Why "what works" won't work: evidence-based practice and the democratic deficit in educational research. Educational Theory, 57(1), 1-22. https://doi.org/10.1111/j.17415446.2006.00241.x

Campbell, D.T. y Stanley, J.C. (1995). Diseños Experimentales y Cuasi-experimentales en la Investigación Social. Amorrortu.

Canabal, C., García, M. y Margalef, L. (2018). La reflexión dialógica en la formación inicial del profesorado. Construyendo un marco conceptual. Perspectiva Educacional, 56(2), 28-50. https//doi.org/10.4151/07189729-Vol.56-Iss.2-Art.496

Cañal, P. (1987). Un enfoque curricular basado en la investigación. Investigación en la Escuela, 1, 43-50. https://bit.ly/3dUkkjv

Elliot, J. (2001). Making evidence-based practice educational. British Educational Research Journal, 27(5), 555574. https://doi.org/10.1080/01411920120095735

Flick, U. (2014). La gestión de la calidad en investigación cualitativa. Morata.

García Gómez, R. J. (2006). Innovación, cultura y poder en las instituciones educativas. CIDE.

Giroux, H. A. (2013). La pedagogía crítica en tiempos oscuros. Praxis Educativa, 17(2), 13-26.

Hammersley, M. (2001). On 'Systematic' Reviews of Research Literatures: A 'narrative' response to Evans \& Benefield. British Educational Research Journal, 27(5), 543-554. https://doi.org/10.1080/01411920120095726

Hammersley, M. y Atkinson, P. (1994). Etnografía. Métodos de investigación. Paidós.

Harris, J. (2007). Enhancing Evolution: The Ethical Case for Making Better People. Princeton University Press.

Iglesias Martínez, M. J., Lozano Cabezas, I., y Roldán Soler, I. (2018). La calidad e innovación educativa en la formación continua docente: un estudio cualitativo en dos centros educativos. Revista Iberoamericana de Educación, 77(1), 13-34. https://doi.org/10.35362/rie7713090

Losada Iglesias, D., Correa Gorospe, J. M. y Fernández Olaskoaga, L. (2017). El impacto del modelo «un ordenador por niño» en la Educación Primaria: Un estudio de caso. Educación XX1, 20(1), 339-361, https://doi.org/10.5944/educXX1.11888

López-Gil, M. y Bernal Bravo, C. (2016). La cultura digital en la escuela pública. Revista Interuniversitaria de Formación del Profesorado, 30(1),103-110. https://bit.ly/3eB7fuB

Martínez Bonafé, J. (2021, 13 de abril). A propósito del cambio curricular que propone Ministerio. El Diario de la Educación. https://bit.ly/3aL519s

Mills, A. J., Durepos, G. \& Wiebe, E. (eds.) (2009). Encyclopedia of Case Study Research (Vols. 1 y 2). SAGE Publications.

Oliver, M. \& Conole, Gr. (2003). Evidence-based practice and e-learning in Higher Education: can we and should we? Research Papers in Education, 18(4), 385-397. http://dx.doi.org/10.1080/0267152032000176873

Ragin, C. \& Becker, H. (eds.) (1992). What is a Case: Exploring the Foundations of Social Enquiry. Cambridge University Press. 
Rodríguez Martínez, C. y Vázquez Recio, R. (2002). Viaje a la escuela rural de Bolonia. En M. Muñoz-Repiso Izaguirre y F. Javier Murillo (Coords.), La mejora de la escuela: un cambio de mirada (pp. 263-289). Ministerio de Educación Cultura y Deporte, Secretaría General Técnica/Octaedro.

Santos, B. S. (2009). Una epistemología del sur: la reinvención del conocimiento y la emancipación social. Siglo XXI Editores.

Santos, B. S. (2010). Descolonizar el saber, reinventar el poder. Ediciones Trilce.

Santos, B. S. (2019). Justicia entre Saberes. Epistemologias del Sur contra el epistemicidio. Morata.

Sego, K., Granados Romero, J. M., Lázaro, M., y Fernández-Larragueta, S. (2017). La formación del profesorado para un uso innovador de las TIC: un estudio de caso en la Educación Obligatoria en la provincia de Almería. Profesorado. Revista de Currículum y Formación de Profesorado, 21(4), 241-258. https://bit.ly/3gROYMa

Simons, H. (1996). El enfoque de estudio de casos en el proyecto sobre la enseñanza de ciencias, matemáticas y tecnología (SMTE) de la OCDE. Revista de Educación, 310, 173-185. https://bit.ly/3eCQVJR

Simons, H. (2011). El estudio de caso: teoría y práctica. Morata.

Stake, R. (1998). Investigación con estudio de casos. Morata.

Stake, R. (2006). Multiple Case Study Analysis. Guilford Press.

Stenhouse, L. (1978). Case Study and case records: toward a contemporary history of education. British Educational Research Journal, 4(2), 21-39. https://doi.org/10.1080/0141192780040202

Stenhouse, L. (1984). Investigación y desarrollo del currículum. Morata.

Stoecker, R. (1991). Evaluating and Rethinking the Case Study. The Sociological Review, 39(1), 88-112. https://doi.org/10.1111/j.1467-954X.1991.tb02970.x

Travers, M. (2006). Qualitative Research Through Case Studies. SAGE Publications.

Vázquez Recio, R. (2011). Enseñanza para la comprensión: El caso de la escuela rural de Bolonia (Cádiz, España). Revista Iberoamericana de Educación, 52(1), 183-202. https://doi.org/10.35362/rie570492

Vázquez Recio, R. y Angulo Rasco, J. F. (2003). Introducción a los estudios de casos: los primeros contactos con la investigación etnográfica. Ediciones Aljibe.

Walker, R. (1983). La realización de estudios de casos en educación. Ética, teoría y procedimientos. En W.B. Dockrell y D. Hamilton (comps.), Nuevas reflexiones sobre la investigación educativa (pp. 42-82). Narcea.

Walker, R. (1989). Métodos de Investigación para el Profesorado. Morata.

Williamson, B. (2019, 1 de febrero). Education for the robot economy. Code acts in education. https://bit.ly/3eAHs5U

Yin, R. K. (2004). The Case Study Anthology. SAGE Publications.

Yin, R. K. (2011). Applications of Case Study Research. SAGE Publications.

Yin, R. K. (2018). Case study research and applications: design and methods. SAGE Publications.

Zeichner, K. (1993). El maestro como profesional reflexivo. Cuadernos de pedagogía, 220, 44-49.

Zeichner, K. y Flessner, R. (2009). Educating teachers for critical education. En M. Apple, W. Au y L. Gandin, (eds.), The Routledge International handbook of critical education (pp. 296-311). Routledge. 